\title{
A Virtual Environment for Distance Learning
}

\author{
Creto Augusto Vidal, Antonio José Melo Leite Júnior, Camilo Camilo \\ Almendra, Emanuele Marques dos Santos, Joaquim Pedro Carvalho de \\ Oliveira and João Carlos Silveira Costa Filho \\ Department of Computing,, Universidade Federal do Ceará, Fortaleza, Ceará, Bloco 910 - \\ Caixa Postal 12.166 -60.455-760, Brazil \\ cvidal@lia.ufc.br
}

Keywords: collaborative learning, distance learning, virtual reality

\begin{abstract}
Brazil, such as many other countries, does not have the capacity to train enough teachers to attend the educational needs of the growing population of children. This has a direct impact on the quality of education that the students receive. The low cost and ease of use of semi-immersive virtual reality environments have recently resulted in a rapid expansion of this technology across the world. In this work, we present a low cost distributed virtual reality system for the education of students from grades $\mathrm{K}$ to 12 . The proposed system is based on an architecture that allows efficient data distribution in low bandwidth networks. We believe that virtual schools, based on the proposed virtual reality system, where remote teachers and students can exchange ideas and propose activities, may be an important part of the solution to this problem.
\end{abstract}

\section{INTRODUCTION}

The State of Ceará in North-eastern Brazil has a serious problem of lowlevel academic and pedagogic formal education of a large portion of its board of teachers. This causes a direct impact on the quality of education that the students receive. The situation is very dramatic in the public school system and even worse in small towns in the countryside of the State. The two main causes for this situation are the low salaries and the poor living 
standards offered by the urban centres in the countryside of Ceará. Considering that the socio-economic development of those cities may take decades to occur, it is very unlikely that, in the short run, the students will be taught by well-prepared teachers, using up to date teaching methodologies.

In order to explore the most recent advances in computer technology and telecommunication, a new distance education program was recently proposed. Its main purpose is to provide the opportunity for continuous education of teachers, especially those from small cities far from the state's capital; and it relies heavily on the use of videoconference. Nine videoconference rooms were specified in the initial phase of the program, one located in the state's capital and the others located in eight urban centres in the countryside of the state. Besides short duration courses or discussions about new teaching methodologies, several licensing degree programs and graduate programs are also being prepared for this project. The implementation of these programs needs a telecommunication infrastructure which will be provided by the State Telecommunication Network, the socalled 'information highways', through which will be transmitted text, sound and image data (Pequeno 1998). Many other applications, based on the Internet protocols, can take advantage of the state's 'information highways'. Thus, other distance education initiatives are being proposed since the connection to the Internet opens up the door to access a wide variety of information resources in the whole world.

In this work, we present a distributed virtual reality system, which relies on the State Telecommunication Network, for the education of students from grades $\mathrm{K}$ to 12 . Our system is well aligned with the UNESCO recommendations for the period from 2002 to 2007 (UNESCO 2000), which encourage the creation of virtual educational communities, of virtual schools and of virtual universities in developing countries. It can be adapted to other regions and countries of the world that can benefit from the ICT (Information and Communication Technologies) potentials for education.

This work is organised as follows: in section 2, we present basic concepts of virtual reality systems; in section 3 , we describe the available resources in the proposed virtual environment; in section 4 , we discuss the components of the architecture used in our system; and in section 5, we draw some conclusions.

\section{VIRTUAL REALITY}

The ideal virtual reality model considers virtual reality as the ultimate navigation tool through which one can visualise, manipulate and interact with computers and complex data sets. Thus, virtual reality can be 
understood as the most advanced type of human-computer interface available nowadays (Çapin et al. 1999). This technology relies in the artificial construction of the so-called virtual world where all the elements with which one wants to interact are embodied in a creative representation defined by the virtual world designer. Once the virtual environment is created, the users, or their representations, can start exploring it and interacting with its elements. The form of interaction in the virtual world tries to mimic, as close as possible, the form of interaction one has in the real world, where the five human senses play a major role.

If the virtual environment is designed in such a way that the users actually feel themselves to be a part of it, we say that the system provides the sense of immersion. In order for a system to provide the sense of total immersion, it would have to stimulate vision, hearing, touch, smell and taste. Is it really possible to achieve total immersion? Could someone, for example, sit on the shade of a virtual tree, feel the freshness of the virtual breeze, see the virtual birds flying and listen to them singing, sense the smell of a virtual apple, take a bite of it and appreciate its flavour? Even if it were possible to use a number of specialised hardware to try to reproduce this simple scene, how useful would this hardware be in reproducing other situations from the real world? Is it really worth the effort to strive for transforming a virtual world into the real world?

For a number of important applications it is enough to be in the middle ground. Thus, a lot of research effort was put into developing specialised hardware and the corresponding software to improve the sense of immersion without using all of the human senses. As a consequence of the research and development effort in special hardware for virtual reality, a great number of equipment is offered in the market today. Also, the computer power had to increase in order to perform complex computations in real time.

Considering the other end of the spectrum, there are virtual reality systems where the screen of a video monitor provides only one sensorial stimulus, the visual stimulus. These types of systems rely essentially on computer graphics to model the virtual world and the user representations, the so-called avatars or virtual humans. The real user interacts with the virtual world from, what some authors call, a 'fish tank' point-of-view. These systems are considered to provide no sense of immersion or just a partial sense of immersion. However, the low cost and ease of use of these systems made them experience a very rapid expansion all over the world. These advantages are stimulating more and more people to devise applications for this type of virtual reality technology. Another very important factor for the growth of this 'fish tank' type of virtual reality is the ease of integration of virtual worlds and users over a network. Now, many applications can be hooked together in a distributed system's approach and the complexity and 
usefulness of the virtual environments are limitless. The users, now, can easily share these complex distributed virtual worlds with other remote users and interact with them in this virtual environment. This type of interaction and socialisation among users brings about an emotional content that is a very important fact to be exploited in many areas.

\section{AVAILABLE RESOURCES}

In this virtual environment, there are important resources available to make it possible for the users to interact with each other. They can use voice communication, which is the most natural form of communication, or exchange text messages, which is the most suitable form of communication over low bandwidth networks.

Sometimes, in a virtual classroom, the students and the teachers want to expose some ideas combining text, $3 \mathrm{D}$ graphics, images, and sound. This can be achieved by using Web pages with all these resources embedded. Another option is to prepare the presentation as three-dimensional panels of text, images, sound and animation that can be arranged in the virtual environment and inspected with a VRML browser.

Another important resource that allows sharing of information is the socalled Whiteboard. It simulates a classroom whiteboard by displaying all the drafts or annotations, written on a small tablet, on a whiteboard in the virtual environment. All the students and teachers logged in the system can work cooperatively using this tool.

The virtual environment can be accessed twenty-four hours a day and, even if none of their classmates or teachers is around to interact with, the student can still interact with simuloids (autonomous virtual humans). These simuloids can help the students to accomplish tasks requested by the teacher during class. The users can have access to information available outside the virtual environment by asking some specialised simuloids, which integrate the virtual world with the Web and with other external resources (Vidal et al. 2000). The simuloids can also assume the role of reproduction avatars. For example, if students miss a mathematics class and later logs into the virtual system, they will be informed that the missed class is available in playback mode in the mathematics virtual classroom. There, a simuloid can be asked to reproduce all the discussion that took place during the class.

Although our virtual system represents a virtual school, it is composed of several specialised virtual worlds, which represent specialised rooms. For example, they can represent study rooms for specific subjects. They can represent training classrooms for continuous education of the schoolteachers, functioning as an alternative to the videoconference based training rooms 
implemented by the Ceará State's Government. They can also represent rooms for simulation of a teacher's office hours, where the student can clear doubts or exchange opinions with the teacher about common interest subjects. The number of specialised virtual worlds that can be incorporated into the system is constrained only by the limits of our imagination and, of course, by the limits of the available hardware.

\section{THE ÁRVORE ARCHITECTURE}

ÁRVORE is a generic architecture, developed so that multi-user virtual reality environments and multimedia resources can be made available even over low bandwidth computer networks. Several client applications provide the necessary means for the user to interact with characters controlled by other users or by computers inside the virtual reality environment. One of the main characteristics of this architecture is the low cost distribution of virtual reality and multimedia elements through specialised replication structures. These types of structures send the elements, upon demand, to be stored locally and in a persistent manner in several predefined components.

Since this is an open architecture, it is possible for the final system to be implemented over several distinct platforms, which are defined according to the specific needs of each provided service. The system is composed of several specialised components, which can be independently implemented. This makes the development of the distributed system easier and, later, its maintenance. The components of the system are divided into two main groups: the server components and the client components.

\subsection{Servers}

The routing server is the basic integration element of the architecture. It controls the information flow among the several components of the virtual environment. The message routing criterion is based upon the service being requested. This defines the way a message is conducted to one or more destinations. The information about the available services is stored, in the routing server, as a persistent list relating the services and their respective locations in the distributed system. The adoption of this routing strategy is essential in warranting the extensibility of the architecture, since the growth of the system occurs by the inclusion of elements followed by a reconfiguration of the list of services. Thus, the functions of these new elements can be rapidly incorporated into the system and made available to all the other components of the distributed system. 
The environment server is responsible for the behaviour control of the virtual reality elements of the virtual world, and maintains the state of all relevant virtual structures, i.e., those that present some type of preprogrammed reaction - characters and tri-dimensional objects. In the virtual world, the interaction between a character and an object triggers events, according to a pre-specified behaviour, which are treated by the environment server.

The replication server is responsible for the distribution upon demand of multimedia and virtual reality elements to the clients of the ÁRVORE architecture. In this architecture, however, video and audio streams are not considered as distribution elements, since they would necessarily require communication channels with high bandwidth. In any case, continuous media can still be made available to the set of clients through a simple transfer of its files. The replication server can be considered one of the main responsible for the low cost of the ÁRVORE architecture. It allows for the distribution of multimedia and virtual reality elements in low speed networks, which, until then, were not able to support such an intense traffic using classical solutions (Sgouros 1999).

The validation server provides session management for the virtual environment, controlling the users' access to the several available resources. The $\log$ server is responsible for the documentation of the overall functioning of the distributed system.

\subsection{Clients}

The users, through the interaction client, access the set of available services in the virtual reality environment. This client is a virtual reality user interface that renders the tri-dimensional structures of the system, taking into consideration the avatars' characteristics and their positions in the virtual environment. Hence, a certain user can interact with other users as well as with objects of the virtual world in which he is immersed.

Simuloids can be implemented as computer-controlled clients, which integrate external applications with the virtual reality environment. Thus, avatars and other simuloids can interact with a given simuloid in order to use, implicitly, the external applications' services that it makes available. To make these interactions as close as possible to those of a human being, it is necessary to establish a pre-programmed behaviour for the simuloid (Çapin et al. 1999).

The management client interacts with certain components of the ÁRVORE architecture. One of its responsibilities is the configuration of the following services: maintenance of the list of services provided by the routing server, maintenance of the access policies contained in the validation 
server, and inclusion of new elements in the replication server. The management client also collects information from several components of the architecture, including the log server, and then generates summaries about the overall functioning of the distributed system. These summaries are dynamically requested and processed in accordance with the system administrators' needs.

\subsection{Model}

The model considered in the ÁRVORE architecture can easily be extended so that multiple virtual worlds run simultaneously. Several instances of the components foreseen in this architecture can be executed in a number of inexpensive microcomputers connected through the Internet. Thus, this architecture provides efficient communication and interaction in shared virtual reality environments to a great number of users. Each virtual world has its own set of server applications. In order to warrant reliability and availability of the system, it is possible to replicate some of the components or even a complete virtual world.

Despite the intensive use of the Internet as a data transmission medium, the proposed ÁRVORE architecture is a low cost solution to the problem just posed, since, from its conception, it does not rely in high-speed networks.

In order to allow the integration and management of the participants' accesses to the whole set of available virtual worlds, it is necessary to include a central structure that is responsible for the storage of the access privileges of all the users of this integrated virtual reality system. The access to the information contained in this central structure can be carried out directly by the Routing Server of each virtual world. However, a much more interesting solution to this access problem is provided by the partitioned replication process (Singhal and Zyda 1999), which is capable of individually updating each validation server used in the virtual environments. Such replications can be made at specific times when the network traffic is less intense, preventing possible congestion.

\subsection{Access}

The users of the system can be organised in access groups, according to the specific needs of a class in a real school's classroom. There are situations where the access to a specific part of the complete virtual environment might be restricted to certain special groups of users. The access rights information is stored in the central structure. In the ÁRVORE architecture, it seems to be natural that the central structure be integrated with the student's record office 
of an educational institution, which maintains a database with all the information about a student's history (class schedules, courses taken, grades obtained, courses' pre-requisites, etc.). The information contained in this database may determine certain access privileges in the virtual environment.

The user can access the virtual system in three ways. First, the user can connect to any of the available virtual worlds. Second, the user directly logs into the central structure. After the user's identification (username and password), the client application is automatically connected to a particular virtual world according to certain predefined access policies. For example, if the user logs into the system at ten o'clock and, according to the user's schedule, she should be attending a biology class, then the user will be directly connected to the corresponding virtual world. Finally, when the user is already connected to a virtual world, she can automatically move to another virtual world, if permission is granted, by just passing through a specific region of the world. This technique is called Portal (Singhal and Zyda 1999).

\section{CONCLUSION}

Virtual reality in Brazil has experienced a considerable growth in recent years and a number of new ideas are being proposed, implemented and tested by research groups in universities and in research institutes. A very promising area of application for virtual reality in Brazil is distance education. Recently the Brazilian government financed a number of research projects on distance education and an expressive part of those projects involved virtual reality applications.

It is a fact that the network infrastructure of Brazil is growing in a faster pace than the capacity of the country to train enough teachers to attend the educational needs of the growing population of children. Therefore, we can dream of virtual schools, those schools with virtual laboratories, fully equipped with virtual instruments available for cooperative virtual experiments. They will also have virtual classrooms, where remote teachers and students can exchange ideas and propose activities. Such a dream is not only for children of small communities in remote areas of the country, but, perhaps, also the only hope for social justice and a way forward for those currently excluded from society. 


\section{REFERENCES}

Çapin, T., Pandzic, I., Magnenat-Thalmann, N. and Thalmann, D. (1999) Avatars in Networked Virtual Environments. John Wiley \& Sons, USA.

Pequeno, M. (1998) Infra-estrutura fisica e tecnológica para Rede de Videoconferência do Estado do Ceará. Project sponsored by FINEP (Financiadora de Estudos e Projetos), Brazil. [www.sct.ce.gov.br/fr-infovia.htm].

Sgouros, N. M. (1999) Involving the Audience in Distributed, Interactive Entertainment Systems. In Proceedings of EG Multimedia' 99 Workshop, Italy.

Singhal, S. and Zyda, M. (1999) Networked Virtual Environments - Design and Implementation. Addison-Wesley, USA.

UNESCO (2000) Hundred and sixtieth Session of Executive Board, 160 EX/17. United Nations Educational, Scientific and Cultural Organization., pp. 11-12. [unesdoc.unesco.org/ulis].

Vidal, C., Leite Jr, A., Almendra, C., Santos, E. and Oliveira, J. (2000) Uma Proposta de Integração de Ferramentas Externas a Ambientes Virtuais através Aplicações Mediadoras e Simulóides. In Proceedings of $3^{\text {rd }}$ Workshop on Virtual Reality, Brazilian Computer Society, Gramado - Brazil, October, pp. 131-142.

\section{BIOGRAPHY}

Creto Vidal is a professor of computer science; his current research interests are computer graphics and virtual reality applications to distance education. He is currently the coordinator of three government-sponsored projects investigating the use of networked virtual environments for training and education (www.lcg.dc.ufc.br/projects).

Melo Leite Júnior is a professor of computer graphics; his current research focus on the applications of networked virtual environments in education and training.

Camilo Almendra, Emanuele Marques, Joaquim Oliveira and João Costa Filho are research assistants in computer graphics and networked virtual reality. 